\title{
Uptake of microplastics and related health effects: a critical discussion of Deng et al., Scientific reports 7:46687, 2017
}

\author{
Albert Braeuning ${ }^{1}$ \\ Received: 12 November 2018 / Accepted: 21 November 2018 / Published online: 23 November 2018 \\ c) Springer-Verlag GmbH Germany, part of Springer Nature 2018
}

Contamination of food with microplastics has recently gained increasing public awareness, based on the detection of small plastic particles in different types of foodstuff as well as in human feces. The European Food Safety Agency (EFSA) has identified major data gaps with respect to microplastic exposure and health effects potentially associated with the oral uptake of such particles (EFSA 2016). According to the literature review by EFSA, previous studies indicate that a very small fraction of particles not exceeding a size of $1.5 \mu \mathrm{m}$ may cross the mammalian intestinal barrier to be subsequently distributed to other tissues. In a recent paper, Yongfeng Deng and colleagues have analyzed the uptake of $5 \mu \mathrm{m}$ and $20 \mu \mathrm{m}$ polystyrene particles in mice and have monitored exposure-associated consequences with a focus on energy and lipid metabolism. The authors conclude that their data suggest widespread health risks of exposure to microplastic particles (Deng et al. 2017).

Elucidation of biological responses to microplastics is an important and timely issue. Unfortunately, the above study by Deng et al. (2017) suffers from drawbacks which should be taken into account when interpreting the data: potential adversity of alterations in the levels of intermediary metabolites, as presented here, is difficult to evaluate. Histological data might be easier to construe, but the occurrence of liver inflammation and hepatic lipid accumulation in livers of microplastic-treated mice, as claimed by the authors, cannot be unequivocally judged based on the histological images provided. Issues with the quality and interpretation of histopathological analyses in a previous publication from the same group have been recently highlighted by others (Baumann et al. 2016) and are therefore not discussed further here. Very small variations of the results of biochemical

Albert Braeuning

Albert.Braeuning@bfr.bund.de

1 Department of Food Safety, German Federal Institute for Risk Assessment, Max-Dohrn-Str. 8-10, 10589 Berlin, Germany measurements, e.g. as shown in Fig. 4 of the Deng et al. (2017) study, might sow doubts with respect to the expected biological variance of data for in vivo studies with $n=5$ per group. In summary, it appears questionable whether the conclusion of health effects by microplastic particles is sufficiently supported by the data provided.

With respect to gastrointestinal uptake of the particles, closer inspection of the data reveals incongruities which question data credibility: the authors have administered daily doses of $0.1 \mathrm{mg}$ of the fluorescent particles (either $5 \mu \mathrm{m}$ or $20 \mu \mathrm{m}$ in diameter) in $0.5 \mathrm{ml}$ of liquid per animal. Particle tissue concentrations were measured in different organs and are presented as $\mathrm{mg}$ particles per $\mathrm{g}$. According to the $Y$ axis labeling in Fig. 2 of the paper, the reference is per $\mathrm{g}$ body weight (bw); nonetheless, it may be assumed that the authors intended to refer to $g$ organ weight here. Regardless the reference value (organ or body weight), however, the presented data cannot have resulted from the experimental protocol described: for example, a total cumulative dose of $0.4 \mathrm{mg}$ particles has been received by animals sacrificed after 4 days of treatment $(4 \times 0.1 \mathrm{mg})$. The liver particle burden, as given in Fig. 2A for the $20 \mu \mathrm{m}$ particle, is approximately $0.5 \mathrm{mg} / \mathrm{g}$ body or organ weight. Using the author's data on body and liver weights of the animals at the beginning of the study (Table $\mathrm{S} 1$ of the paper: $\sim 36 \mathrm{~g}$ body weight, $\sim 1.7 \mathrm{~g}$ liver weight), one can calculate a total particle burden of $0.5 \mathrm{mg} / \mathrm{g} \mathrm{bw} * 36 \mathrm{~g} \mathrm{bw}=18 \mathrm{mg}$ when referring to body weight, or $0.5 \mathrm{mg} / \mathrm{g} \times 1.7 \mathrm{~g}=0.85 \mathrm{mg}$ when referring to organ weight. Both values considerably exceed the total administered particle dose. The latter (and lower) value of $0.85 \mathrm{mg}$ particles still exceeds $200 \%$ of the received dose and that number does not include the substantial amounts of particles claimed to be present in gut and kidney. Besides the plausibility problem of uptake data as detailed above, high tissue levels of polystyrene microparticles after oral ingestion contradict previous findings on polystyrene microplastic bioavailability as summarized by EFSA (2016). 
Unfortunately, these discrepancies are not discussed in the paper by Deng et al. (2017).

In conclusion, a number of issues prevent the usage of the data from Deng et al. (2017) for the estimation of gastrointestinal microplastic uptake and potential health risks associated with the oral uptake of microplastics. Further research is needed to close existing knowledge gaps on microplastic uptake and to allow for a proper assessment of possible health risks to humans.

\section{References}

Baumann L, Schmidt-Posthaus H, Segner H, Wolf JC (2016) Comment on "Uptake and Accumulation of Polystyrene Microplastics in zebrafish (Danio rerio) and Toxic Effects in Liver". Environ Sci Technol 50:12521-12522

Deng Y, Zhang Y, Lemos B, Ren H (2017) Tissue accumulation of microplastics in mice and biomarker responses suggest widespread health risks of exposure. Sci Rep 7:46687

EFSA Panel on Contaminants in the Food Chain (2016) Presence of microplastics and nanoplastics in food, with particular focus on seafood. EFSA J 14:4501 